\title{
Improving Breakdown Voltage for a Novel SOI LDMOS with a Lateral Variable Doping Profile on the Top Interface of the Buried Oxide Layer
}

\author{
Jingjing Jin,, Shengdong Hu, ${ }^{1,2}$ Yinhui Chen, ${ }^{1}$ Kaizhou Tan, ${ }^{2}$ Jun Luo, ${ }^{2}$ Feng Zhou, \\ Zongze Chen, ${ }^{1}$ and Ye Huang ${ }^{1}$ \\ ${ }^{1}$ College of Communication Engineering, Chongqing University, Chongqing 400044, China \\ ${ }^{2}$ National Laboratory of Analogue Integrated Circuits, No. 24 Research Institute of China Electronics Technology Group Corporation, \\ Chongqing 400060, China \\ Correspondence should be addressed to Shengdong Hu; hushengdong@hotmail.com
}

Received 13 January 2015; Revised 27 May 2015; Accepted 28 June 2015

Academic Editor: Wai Tung Ng

Copyright ( 2015 Jingjing Jin et al. This is an open access article distributed under the Creative Commons Attribution License, which permits unrestricted use, distribution, and reproduction in any medium, provided the original work is properly cited.

In order to achieve a high breakdown voltage (BV) for the SOI (Silicon-On-Insulator) power device in high voltage ICs, a novel high voltage n-channel lateral double-diffused MOS (LDMOS) with a lateral variable interface doping profile (LVID) placed at the interface between the SOI layer and the buried-oxide (BOX) layer (LVID SOI) is researched. Its breakdown mechanism is investigated theoretically, and its structure parameters are optimized and analyzed by 2D simulation software MEDICI. In the high voltage blocking state, the high concentration ionized donors in the depleted LVID make the surface electric field of SOI layer $\left(E_{S}\right)$ more uniform and enhance the electric field of BOX layer $\left(E_{I}\right)$, which can prevent the lateral premature breakdown and result in a higher BV. Compared with the conventional uniformly doped (UD) SOI LDMOS, $E_{I}$ of the optimized LVID SOI LDMOS is enhanced by $79 \%$ from $119 \mathrm{~V} / \mu \mathrm{m}$ to $213 \mathrm{~V} / \mu \mathrm{m}$, and BV is increased by $33.4 \%$ from $169 \mathrm{~V}$ to $227 \mathrm{~V}$. Simulations indicate that the method of LVID profile can significantly improve breakdown voltage for the SOI LDMOS.

\section{Introduction}

SOI technology has been widely used in high voltage ICs due to its advantages such as superior isolation, high speed, and low loss [1]. However, SOI power devices suffer from a low vertical BV. A feasible way to increase the BV is to enhance the dielectric layer field (ENDIF), and several new structures have been proposed by ENDIF, in which introducing interface charges is effective and attractive [2]. The variable doping profile is a method of introducing interface charges which was firstly proposed to avoid high voltage breakdown for planar junctions in 1985 [3]. The approach of designing and implementing the linear doping profile on thin SOI for lateral high voltage devices was presented in 1995 [4]. A submicron thin film SOI LDMOS with the variable doping profile and numerical modeling of linear doping profiles were proposed in 1996 and 1999, respectively [5, 6]. After 2000, several new SOI devices structures with linear doping profile were proposed [7-11].

In this paper, a novel SOI device with a LVID profile (LVID SOI LDMOS) is designed. The doping profile of drift region is a combination of linear variable doping and uniform doping in vertical dimension, which is different from the above-mentioned structures. The influence of the parameters on $\mathrm{BV}$ and the specific on-resistance $\left(R_{\mathrm{on}, \mathrm{sp}}\right)$ is presented. The simulated results indicate that the method of LVID profile can significantly improve BV compared with UD profile.

\section{Structure and Mechanism}

The cross-section of LVID SOI LDMOS is illustrated in Figure 1(a). The LVID profile is placed at the interface between the SOI layer and the BOX layer, the thickness of which is expressed as $t_{\text {lvid }}$. The lateral doping concentration 


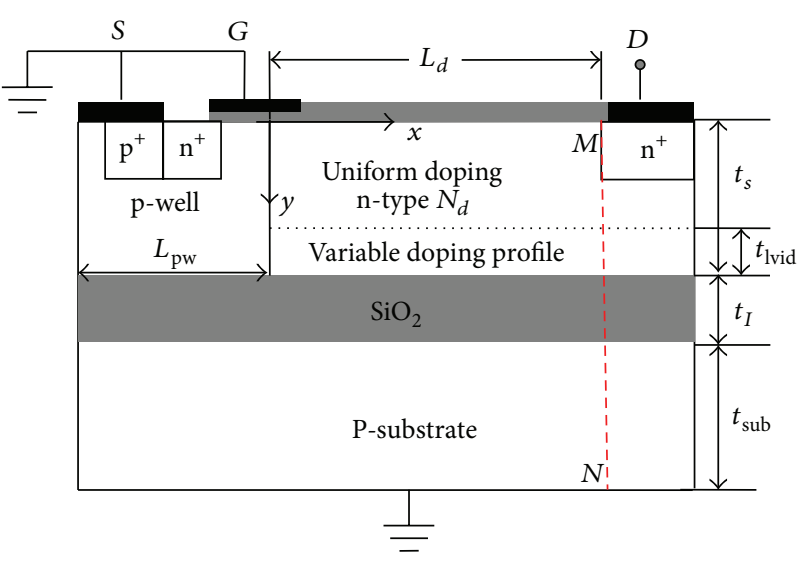

(a)

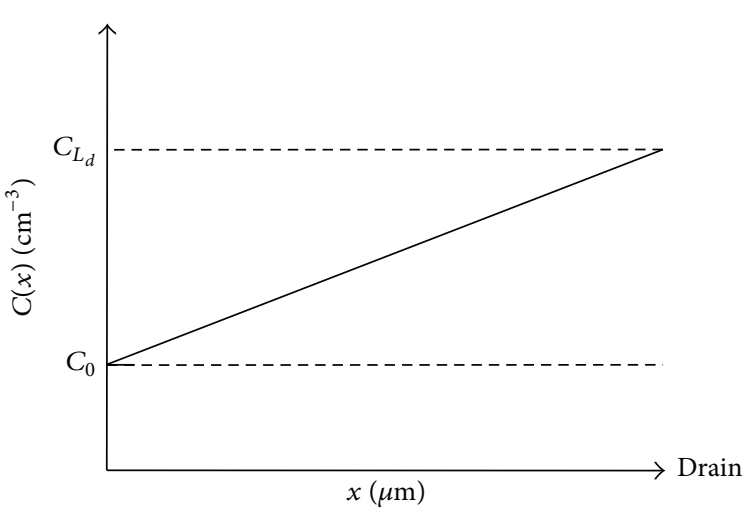

(b)

FIgURE 1: LVID SOI LDMOS. (a) Schematic cross-section; (b) the doping concentration of the LVID profile.

of the LVID profile is shown in Figure 1(b), which can be expressed as

$$
C(x)=G x+C_{0}
$$

where $x$ is the distance away from source, $G$ is the linear graded coefficient which determines the slope of the profile, $C(x)$ is the doping concentration of LVID profile, and $C_{0}$ is the doping concentration at $x=0, y=t_{s}$. Here, $t_{s}$ is the thickness of the SOI layer and $N_{d}$ is the doping concentration of uniform doping section.

According to Figure 1(b) and (1),

$$
G=\frac{C_{L_{d}}-C_{0}}{L_{d}},
$$

where $C_{L_{d}}$ is the doping concentration at $x=L_{d}, y=t_{s}$. Since $C_{0}$ is much less than $C_{L_{d}}$, it is ignored in the above expression. $G$ is proportional to $C_{L_{d}}$.

The device mechanism at breakdown can be explained as follows. When a high positive voltage $V_{d}$ is applied to the drain while the source, gate, and substrate are grounded, the LVID profile will be depleted and high concentration ionized donors are accumulated at the top interface of BOX layer just as shown in Figure 2, and due to the LVID profile, the concentration of ionized donors in LVID profile will linearly increase from source to drain. According to the theory of ENDIF, the interface charges can not only increase the electric field in BOX layer but modulate the surface electric field of drift region [2]. The modulation effect is that the surface electric field peaks at source and drain are decreased and the surface electric field in the middle drift region is improved. Thus LVID profile should prevent the lateral premature breakdown. At the same time, because the vertical breakdown voltage is sustained by the BOX layer, the improved $E_{I}$ can significantly enhance the vertical BV. The influences of the LVID profile on the breakdown performance of SOI LDMOS transistor will be analyzed and compared with UD SOI LDMOS in Section 3. The parameters used in the simulations are detailed in Table 1.

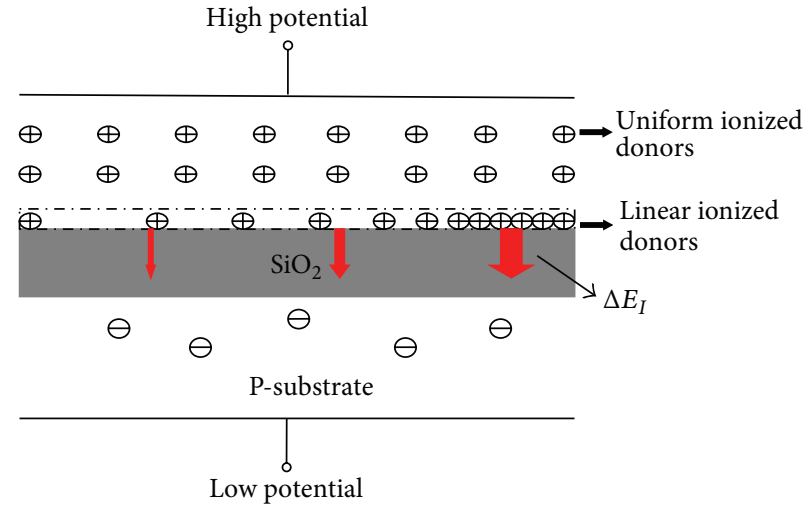

FIGURE 2: Device mechanism of the LVID SOI LDMOS.

TABLE 1: Device parameters used in the simulations.

\begin{tabular}{lc}
\hline Parameter & Value \\
\hline Thickness of the SOI layer, $t_{s}(\mu \mathrm{m})$ & 2 \\
Thickness of the buried oxide layer, $t_{I}(\mu \mathrm{m})$ & 1 \\
Thickness of P-substrate, $t_{\text {sub }}(\mu \mathrm{m})$ & 2 \\
Thickness of the LVID profile, $t_{\text {lvid }}(\mu \mathrm{m})$ & $0.1-2$ \\
Length of n-type drift region, $L_{d}(\mu \mathrm{m})$ & 10 \\
Length of the p-well, $L_{\mathrm{pw}}(\mu \mathrm{m})$ & 5 \\
Concentration of P-substrate, $N_{\text {sub }}\left(\mathrm{cm}^{-3}\right)$ & $8 \times 10^{14}$ \\
Concentration of p-well, $N_{\mathrm{pw}}\left(\mathrm{cm}^{-3}\right)$ & $3 \times 10^{16}$ \\
Concentration of uniform doping in drift & Optimized \\
region, $N_{d}\left(\mathrm{~cm}^{-3}\right)$ & Optimized \\
The slope of the LVID profile, $G\left(\mathrm{~cm}^{-4}\right)$ &
\end{tabular}

\section{Results and Discussion}

Figure 3 is the electrical performances at breakdown of the two devices whose structure parameters are optimized with $t_{\text {lvid }}=0.1 \mu \mathrm{m}$. UD SOI LDMOS reaches the maximum BV at $N_{d}=9 \times 10^{15} \mathrm{~cm}^{-3}$, and LVID SOI LDMOS reaches the maximum $\mathrm{BV}$ at $N_{d}=4 \times 10^{15} \mathrm{~cm}^{-3}, G=4.48 \times 10^{20} \mathrm{~cm}^{-4}$. 


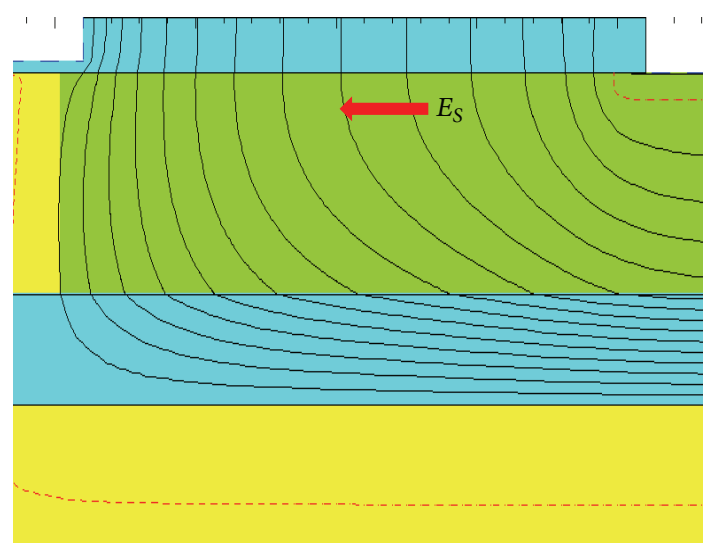

(a)

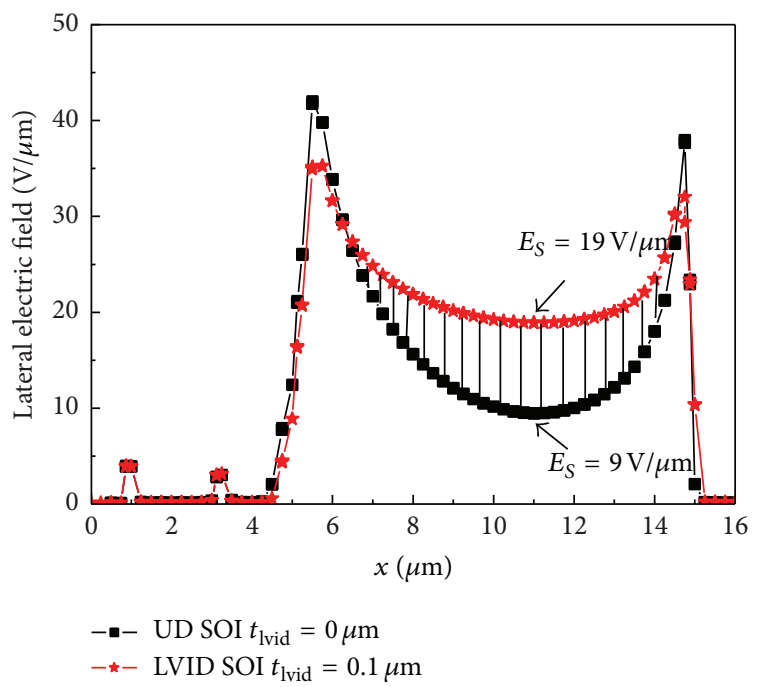

(c)

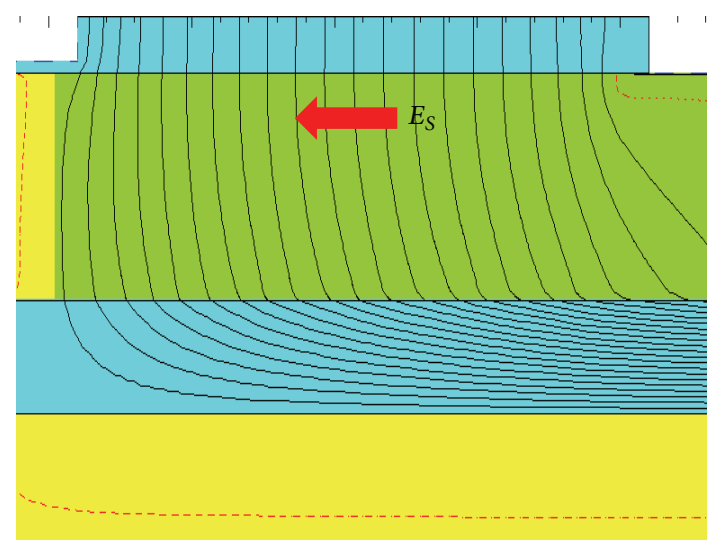

(b)

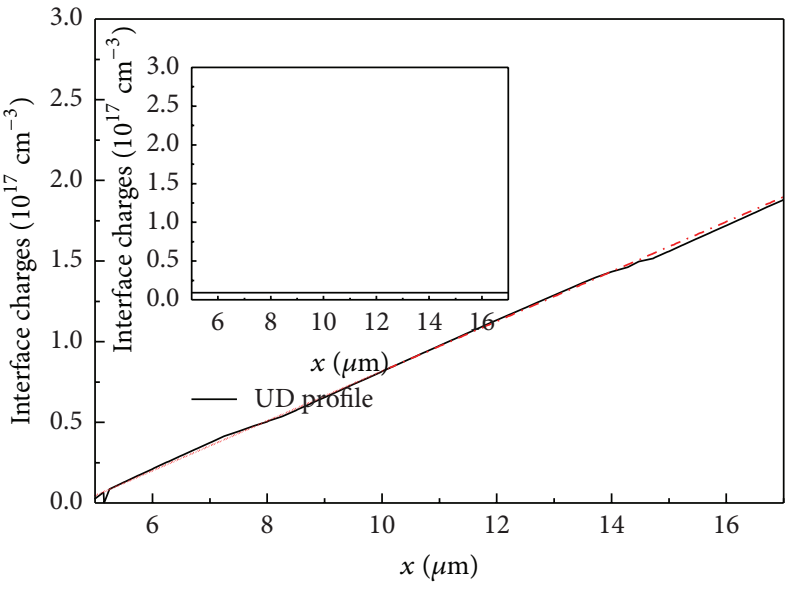

— LVID profile

(d)

FIGURE 3: Distributions of equipotential contours, electric fields, and interface charges at breakdown for the UD and LVID SOI LDMOS. (a) Equipotential contours ( $10 \mathrm{~V} /$ contour) distributions for the UD SOI LDMOS with the optimized $N_{d}=9 \times 10^{15} \mathrm{~cm}^{-3}$; (b) equipotential contours (10 V/contour) distributions for the LVID SOI LDMOS with the optimized $N_{d}=4 \times 10^{15} \mathrm{~cm}^{-3}, G=4.48 \times 10^{20} \mathrm{~cm}^{-4}$. (c) The comparison of the lateral surface electric field distributions at breakdown. (d) Interface charges concentration distributions of LVID profile and UD profile at breakdown.

Figures 3(a) and 3(b) are the equipotential contours distributions at breakdown for the two devices. It can be seen that, compared with the UD SOI LDMOS shown in Figure 3(a), the equipotential contours of the LVID SOI LDMOS shown in Figure 3(b) are more uniform and denser. The surface electric field distributions at breakdown for the two devices are shown in Figure 3(c). It is clear that the $E_{S}$ of LVID SOI LDMOS is optimized and more uniform, and the $E_{S}$ at $x=$ $L_{d} / 2$ is improved by $111 \%$ compared with UD SOI LDMOS which means an increase of lateral $\mathrm{BV}$, and the shadow part is just the improvement value of lateral BV. The optimization of $E_{S}$ and improvement of lateral $\mathrm{BV}$ are the results of the high concentration ionized donors distributed in the LVID profile as shown in Figure 3(d), the inset of which is the lower ionized donors concentration distribution of UD profile.

The vertical electric fields and potentials distributions for the UD and LVID SOI LDMOS at breakdown along line MN (shown in Figure 1(a)) are shown in Figure 4. It is clear that, for the LVID SOI LDMOS, $E_{I}$ is improved by $79 \%$ from $119 \mathrm{~V} / \mu \mathrm{m}$ to $213 \mathrm{~V} / \mu \mathrm{m}$, and $\mathrm{BV}$ is improved by $34.3 \%$ from $169 \mathrm{~V}$ to $227 \mathrm{~V}$, which are both the results of the introduced interface charges in the LVID profile.

Figure 5 shows the dependences of $\mathrm{BV}$ on the $G$ for different $N_{d}$. Simulation results indicate that there is always an optimum concentration slope for different $N_{d}$ to make $B V$ reach the maximum value and the optimum $G$ decreases with the increase of $N_{d}$. The reason is that the total doping concentration of drift region needed for the device breakdown at the maximum BV is certain by RESURF (REduced SURface Field) theory [12]. When $N_{d}$ increases, the doping concentration of LVID profile needed for the maximum BV will decrease, which is proportional to $G$ as defined in (2). So $G$ is inversely proportional to $N_{d}$. Figure 6 shows the dependences of BV on $N_{d}$ for different $G$. Results indicate that there is also always an optimum $N_{d}$ for different $G$ to make $\mathrm{BV}$ reach the maximum value and the optimum $N_{d}$ decreases 


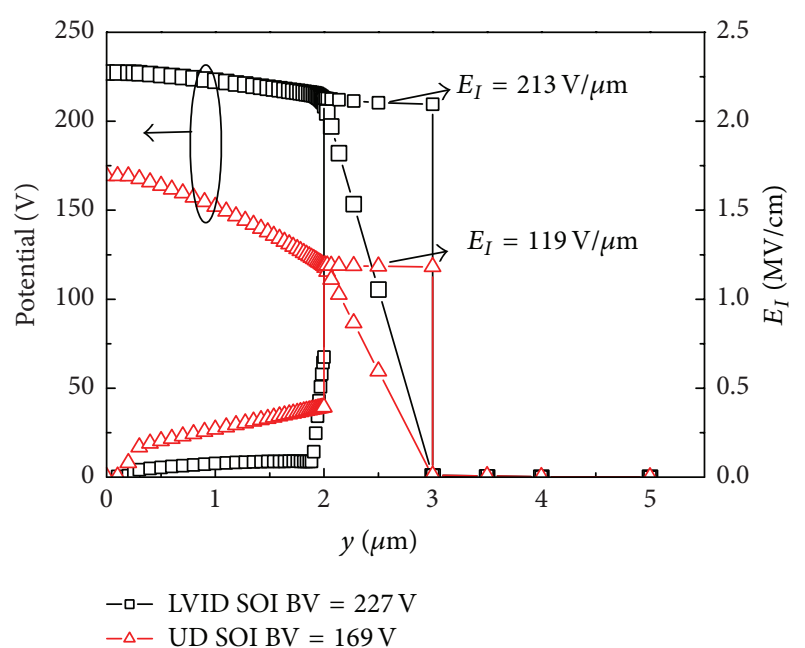

FIGURE 4: Vertical electric fields and potentials distributions at breakdown along line MN for UD SOI LDMOS $\left(N_{d}=9 \times 10^{15} \mathrm{~cm}^{-3}\right)$ and LVID SOI LDMOS $\left(N_{d}=4 \times 10^{15} \mathrm{~cm}^{-3}, G=4.48 \times 10^{20} \mathrm{~cm}^{-4}\right)$.

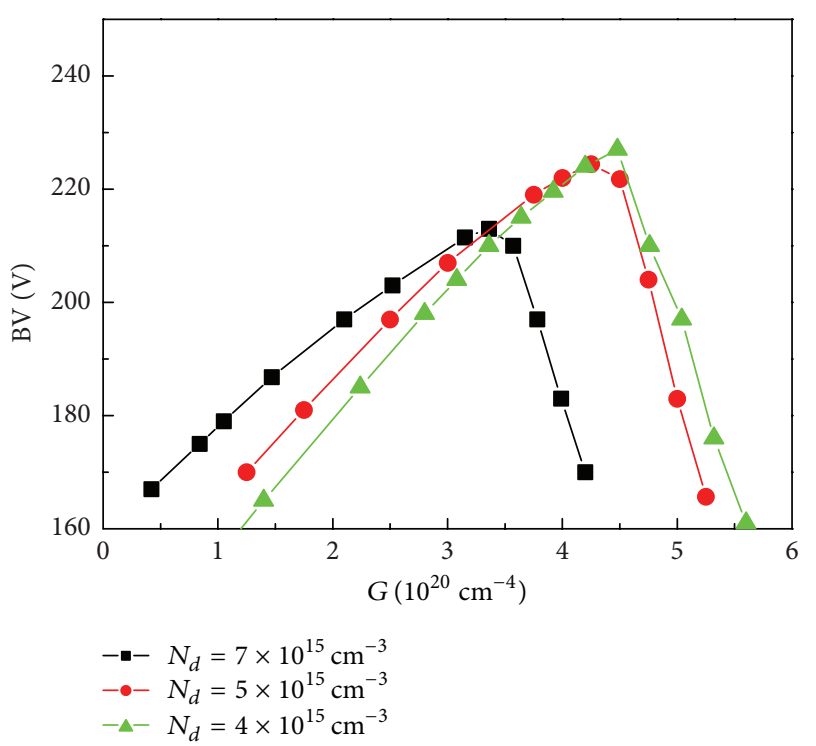

FIGURE 5: Dependences of BV on $G$ with $t_{\text {lvid }}=0.1 \mu \mathrm{m}$.

with the increase of $G$. The results accord with the inverse relationship of $G$ and $N_{d}$.

Figure 7 shows the influence of $t_{\text {lvid }}$ on BV. It can be seen that $G$ is inversely proportional to $t_{\text {lvid }}$, which means the optimum $G$ decreases with the increase of $t_{\text {lvid }}$. This is also because the total doping concentration needed for the maximum BV is certain. Simulations show that when $t_{\text {lvid }}$ is less than $0.5 \mu \mathrm{m}, \mathrm{BV}$ can reach maximum values above $210 \mathrm{~V}$ for different $t_{\text {lvid }}$ and attains $227 \mathrm{~V}$ at $t_{\text {lvid }}=0.1 \mu \mathrm{m}$. When $t_{\text {lvid }}$ is greater than $0.6 \mu \mathrm{m}, \mathrm{BV}$ falls quickly and drops to $184 \mathrm{~V}$ at $t_{\text {lvid }}=1 \mu \mathrm{m}$. BV remains in about $180 \mathrm{~V}$ within $1 \mu \mathrm{m}$ to $2 \mu \mathrm{m}$. It can be concluded that $t_{\text {lvid }}$ has a great influence on BV within $0.5 \mu \mathrm{m}$ and has a little influence on BV when $t_{\text {lvid }}$ is greater than $1 \mu \mathrm{m}$.

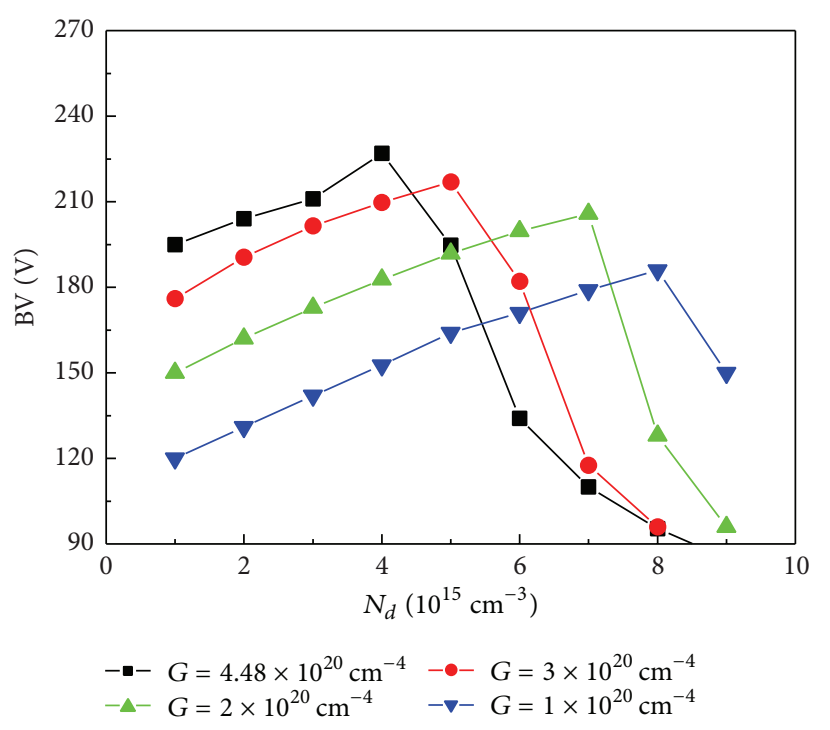

FIgURE 6: Dependences of BV on $N_{d}$ with $t_{\text {lvid }}=0.1 \mu \mathrm{m}$.

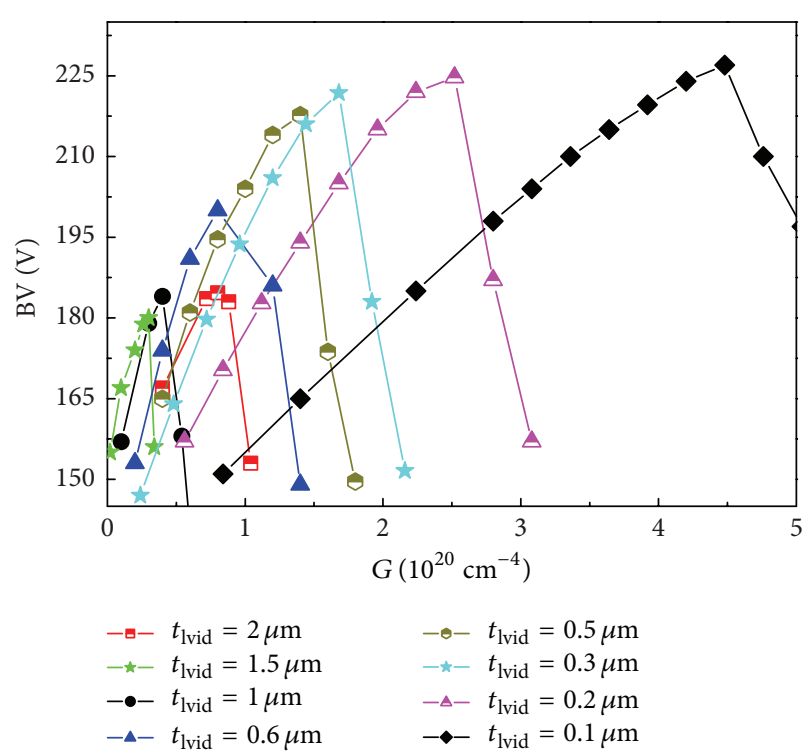

FIgURE 7: The influence of $t_{\text {lvid }}$ on BV.

Figure 8 shows the influences of $t_{\text {lvid }}$ on BV and $R_{\text {on,sp }}$. The results are obtained in the case of the optimized devices for the highest $\mathrm{BV}$ for different $t_{\text {lvid. }}$. It can be seen that $\mathrm{BV}$ and $R_{\text {on,sp }}$ both decrease with the increase of $t_{\text {lvid }}$. Figure of Merit (FOM) is a value calculated by $\mathrm{BV}^{2} / R_{\mathrm{on}, \mathrm{sp}}$ which balances the trade-off between $\mathrm{BV}$ and $R_{\mathrm{on}, \mathrm{sp}}$ [13]. The optimized FOM is $6.22 \mathrm{MW} / \mathrm{cm}^{2}$ at $t_{\text {lvid }}=0.1 \mu \mathrm{m}$ when gate voltage is $15 \mathrm{~V} . R_{\mathrm{on}, \mathrm{sp}}$ is calculated by $L_{d} \times V_{d s} / I_{d s}$ at the linear region of $I-V$ curve and is $8.28 \mathrm{~m} \Omega \cdot \mathrm{cm}^{2}$ at $t_{\text {lvid }}=0.1 \mu \mathrm{m}$.

Figure 9 shows the major fabrication steps for an LVID SOI wafer: (a) implanting arsenic to form the LVID profile region on the bottom of the SOI layer; (b) thermally growing to form the buried oxide layer on the p-type substrate; (c) bonding the SOI layer and the substrate layer; (d) annealing 


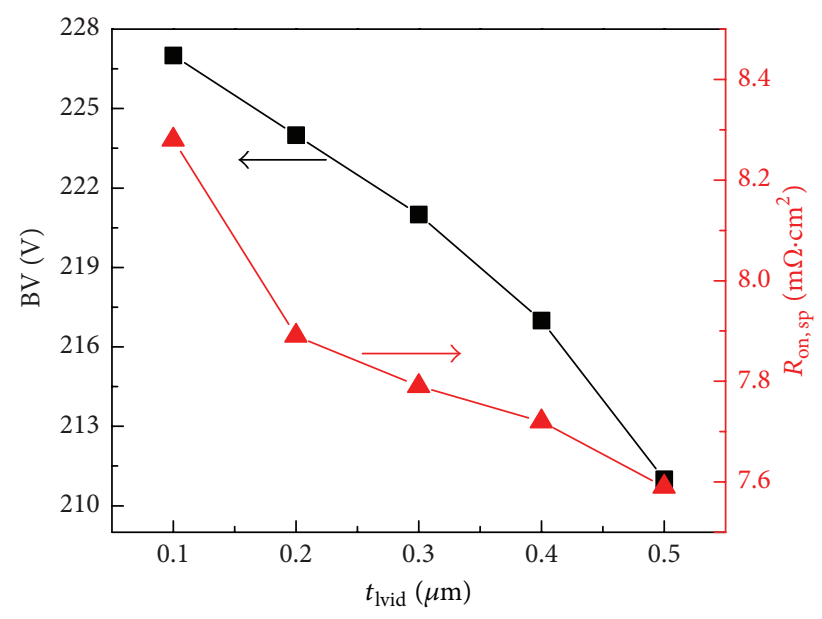

FIgURE 8: The influences of $t_{\text {lvid }}$ on BV and $R_{\text {on,sp }}$.

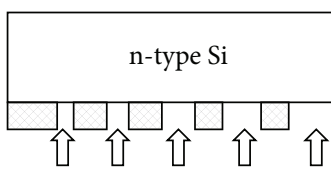

Arsenic implantation

(a)

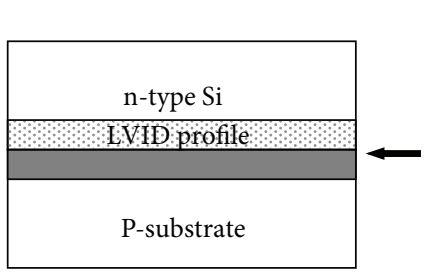

(d)

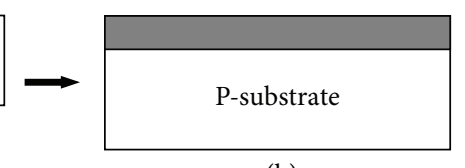

b)

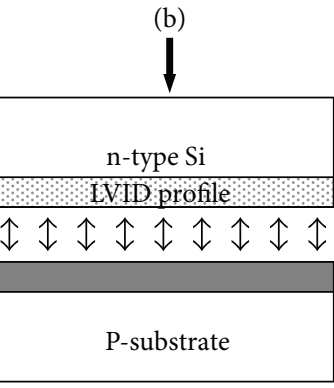

(c)

\footnotetext{
$\square$ Photoresist

$\mathrm{n}^{+}$silicon

$\square \mathrm{SiO}_{2}$
}

FIGURE 9: Major fabrication steps of LVID profile.

the wafer and then thinning and polishing the wafer. The LVID profile can be achieved by using the lateral variation doping technique $[4,9]$. It can be seen that only an additional process of arsenic implanting before bonding is needed to form LVID SOI wafer. Other fabrication processes are fully compatible with conventional CMOS/SOI technology.

\section{Conclusion}

The LVID SOI high voltage device structure is proposed. The proposed structure increases greatly the BV because of the modulation effect of interface charges on the electric fields in the BOX layer and drift region. Compared with UD SOI LDMOS, $E_{I}$ and BV of LVID SOI LDMOS are improved by $79 \%$ and $33.4 \%$, respectively, which proves that the LVID profile is an effective method to improve the BV for SOI high voltage devices.

\section{Conflict of Interests}

The authors declare that there is no conflict of interests regarding the publication of this paper.

\section{Acknowledgments}

This work is supported by the National Natural Science Foundation of China (Grant no. 61404014) and the Fundamental Research Funds for the Central Universities, China (Grant no. 106112014CDJZR168801).

\section{References}

[1] H. Tomita, H. Eguchi, S. Kijima et al., "Wide-voltage SOIBiCDMOS technology for high-temperature automotive applications," in Proceedings of the 23rd International Symposium on Power Semiconductor Devices and ICs (ISPSD '11), pp. 28-31, May 2011.

[2] B. Zhang, Z. Li, S. Hu, and X. Luo, "Field enhancement for dielectric layer of high-voltage devices on silicon on insulator," IEEE Transactions on Electron Devices, vol. 56, no. 10, pp. 23272334, 2009.

[3] R. Stengl and U. Gosele, "Variation of lateral doping-a new concept to avoid high voltage breakdown of planar junctions," in Proceedings of the International Electron Devices Meeting, pp. 154-157, 1995.

[4] T. M. L. Lai, J. K. O. Sin, M. Wang, V. M. C. Poon, and P. K. Ko, "Implemention of linear doping profiles for high voltage thin-film SOI devices," in Proceedings of the 7th International Symposium on Power Semiconductor Devices and ICs (ISPSD '95), pp. 315-320, IEEE, Yokohama, Japan, May 1995.

[5] A. K. Paul, Y. K. Leung, J. D. Plummer et al., "High voltage LDMOS transistors in sub-micron SOI films," in Proceedings of the 8th International Symposium on Power Semiconductor Devices and ICs (ISPSD '96), pp. 89-92, Maui, Hawaii, USA, May 1996.

[6] S. D. Zhang, J. K. O. Sin, T. M. L. Lai, and P. K. Ko, "Numerical modeling of linear doping profiles for high-voltage thin-film SOI devices," IEEE Transactions on Electron Devices, vol. 46, no. 5, pp. 1036-1041, 1999.

[7] J. Roig, D. Flores, I. Cortés, S. Hidalgo, and J. Millán, “Thin film SOI and SOS LDMOS structures with linear doping profile and enlarged field plate," in Proceedings of the 24th International Conference on Microelectronics (MIEL '04), vol. 1, pp. 141-144, IEEE, May 2004.

[8] S. Yang, W. Tseng, and G. Sheu, "Dependence of breakdown voltage on drift length and linear doping gradients in SOI RESURF LDMOS devices," in Proceedings of the 9th International Conference on Electronic Measurement \& Instruments (ICEMI '09), pp. 4-594-4-597, IEEE, Beijing, China, August 2009.

[9] L. J. Wu, W. T. Zhang, M. Qiao, B. Zhang, and Z. J. Li, “SOI SJ high voltage device with linear variable doping interface thin silicon layer," Electronics Letters, vol. 48, no. 5, pp. 287-289, 2012.

[10] Z. Wang, X. Cheng, D. He et al., "Realization of $850 \mathrm{v}$ breakdown voltage LDMOS on Simbond SOI," Microelectronic Engineering, vol. 91, pp. 102-105, 2012.

[11] Y. Li, M. Qiao, Y. Jiang, X. Zhou, W. Xu, and B. Zhang, "Uniform and linear variable doping ultra-thin PSOI LDMOS with n-type buried layer," Electronics Letters, vol. 49, no. 22, pp. 1407-1409, 2013. 
[12] Y. S. Huang and B. J. Baliga, "Extension of resurf principle to dielectrically isolated power devices," in Proceedings of the $3 \mathrm{rd}$ International Symposium on Power Semiconductor Devices and ICs (ISPSD '91), pp. 27-30, April 1991.

[13] B. J. Baliga, "Power semiconductor device figure of merit for high-frequency applications," IEEE Electron Device Letters, vol. 10, no. 10 , pp. $455-457,1989$. 

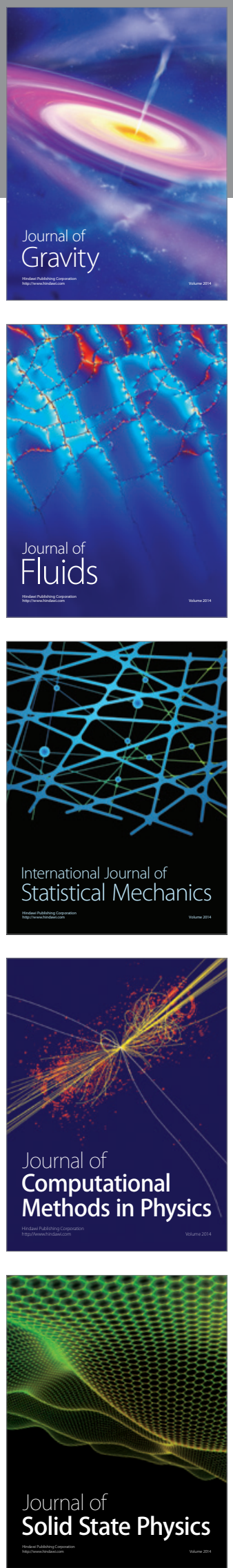

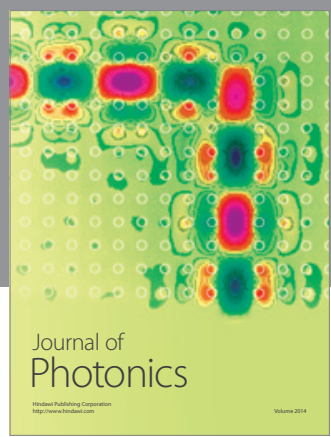

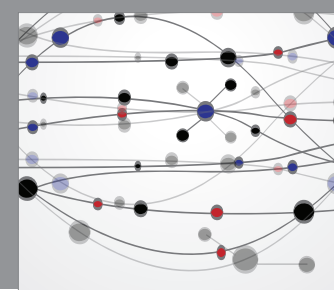

The Scientific World Journal

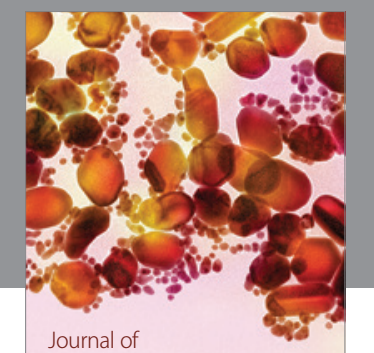

Soft Matter
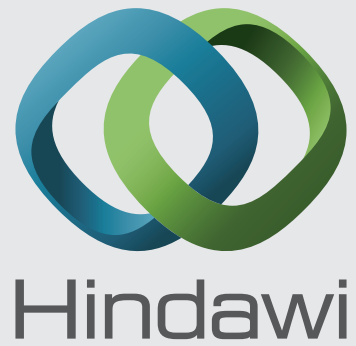

Submit your manuscripts at

http://www.hindawi.com
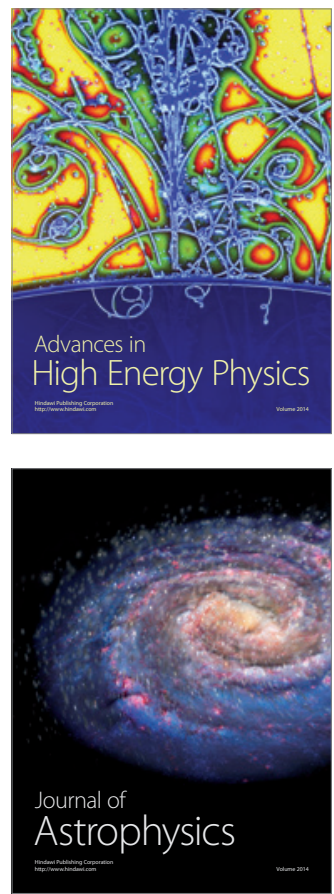
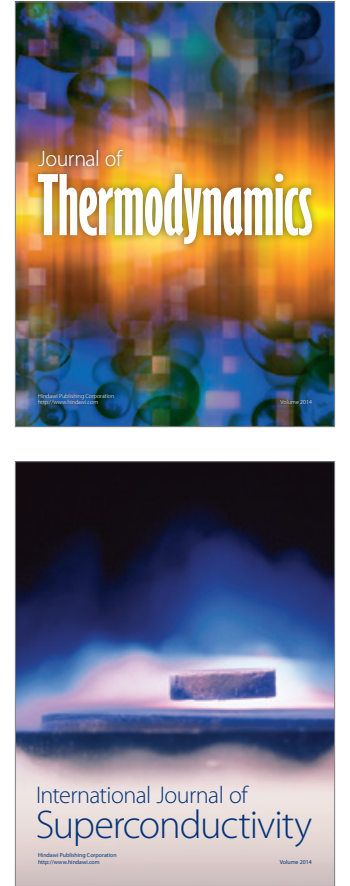
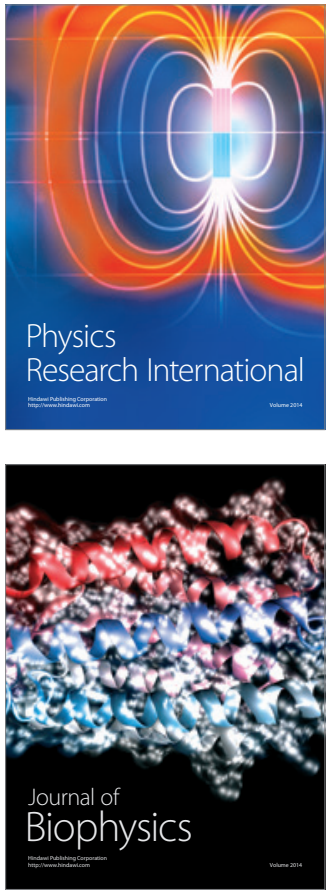
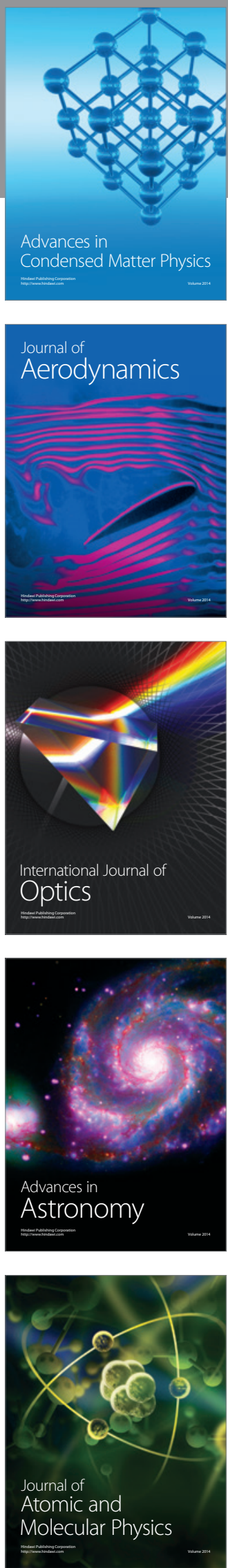